\title{
Error Estimates for Gauss Quadrature Formulas for Analytic Functions
}

\author{
By M. M. Chawla and M. K. Jain
}

1. Introduction. The estimation of quadrature errors for analytic functions has been considered by Davis and Rabinowitz [1]. An estimate for the error of the Gaussian quadrature formula for analytic functions was obtained by Davis [2]. McNamee [3] has also discussed the estimation of error of the Gauss-Legendre quadrature for analytic functions. Convergence of the Gaussian quadratures was discussed by Barrett [4].

The object of the present paper is to derive error estimates for the GaussLegendre and the Gauss-Chebyshev quadrature formulas applied to analytic functions. A few lemmas, which are useful for the derivation of these estimates, have also been proved.

2. The Gauss-Legendre Quadrature Formula. Let $L$ be a closed contour enclosing the interval $[-1,1]$ in the $z$-plane and let the zeros of the Legendre polynomial $P_{n}(t)$ be denoted by $\left\{t_{i}\right\}_{1}{ }^{n}$. On applying the residue theorem to the contour integral

$$
\frac{1}{2 \pi i} \int_{L} \frac{f(z) d z}{(z-t) P_{n}(z)}
$$

we get

$$
f(t)=\sum_{i=1}^{n} \frac{P_{n}(t)}{\left(t-t_{i}\right) P_{n}^{\prime}\left(t_{i}\right)} f\left(t_{i}\right)+\frac{1}{2 \pi i} \int_{L} \frac{f(z) P_{n}(t) d z}{(z-t) P_{n}(z)}
$$

if $f(z)$ is regular within $L$.

Integrating both sides with respect to $t$ over $[-1,1]$ and interchanging the order of integration on the right side, we obtain

$$
\int_{-1}^{1} f(t) d t=\sum_{i=1}^{n} \lambda_{i} f\left(t_{i}\right)+E_{G_{n}}(f) .
$$

This is the Gauss-Legendre quadrature formula of order $n$ over the interval $[-1,1]$. Here

$$
\lambda_{i}=\frac{1}{P_{n}^{\prime}\left(t_{i}\right)} \int_{-1}^{1} \frac{P_{n}(t) d t}{t-t_{i}}
$$

are the weights of the quadrature formula known to be positive. The abscissas $t_{i}$ and the weights $\lambda_{i}$ have been tabulated extensively.

The error of the Gauss-Legendre quadrature formula is given by

$$
E_{G_{n}}(f)=\frac{1}{\pi i} \int_{L} \frac{Q_{n}(z) f(z) d z}{P_{n}(z)}
$$

where we have put

Received January 5, 1966. Revised September 20, 1966 and April 6, 1967. 


$$
Q_{n}(z)=\frac{1}{2} \int_{-1}^{1} \frac{P_{n}(t) d t}{z-t} .
$$

$Q_{n}(z)$, the Legendre function of the second kind, is single valued and analytic in the $z$-plane with the interval $[-1,1]$ deleted.

We introduce the mapping

$$
z=\frac{1}{2}\left(\xi+\xi^{-1}\right), \quad \xi=\rho e^{i \theta} \quad(0 \leqq \theta \leqq 2 \pi) .
$$

This maps the exterior of the unit circle $|\xi|=1$ conformally onto the $z$-plane with the interval $[-1,1]$ deleted. The circle $|\xi|=\rho(\rho>1)$ is mapped onto an ellipse $\varepsilon_{\rho}$ with foci at $z= \pm 1$ and semi-axes $\frac{1}{2}\left(\rho+\rho^{-1}\right)$ and $\frac{1}{2}\left(\rho-\rho^{-1}\right)$.

2.1. A Lemma for $Q_{n}(z)$. The following lemma has been proved in [5, Lemma 12.4.6].

Lemma 1. For $z \in \varepsilon_{\rho}$

$$
Q_{n}(z)=\sum_{k=n+1}^{\infty} \frac{\sigma_{n k}}{\xi^{k}}
$$

where

$$
\left|\sigma_{n k}\right| \leqq \pi, \quad n=0,1,2, \cdots, \quad k=n+1, n+2, \cdots .
$$

We improve upon the above lemma as follows:

Lemma 2. For $z \in \varepsilon_{\rho}$

$$
Q_{n}(z)=\sum_{k=n+1}^{\infty} \frac{\sigma_{n k}}{\xi^{k}}
$$

where

$$
\left|\sigma_{n k}\right| \leqq 2, \quad n=0,1,2, \cdots, \quad k=n+1, n+3, \cdots .
$$

Proof. Following Davis [5, p. 311], setting $t=\cos \theta$ and transforming (5) to the $\xi$-plane,

$$
Q_{n}(z)=\xi^{-1} \int_{0}^{\pi} \frac{P_{n}(\cos \theta) \sin \theta d \theta}{1-2 \xi^{-1} \cos \theta+\xi^{-2}}
$$

Now

$$
\frac{\sin \theta}{\xi}\left[1-2 \xi^{-1} \cos \theta+\xi^{-2}\right]^{-1}=\sum_{m=1}^{\infty} \frac{\sin (m \theta)}{\xi^{m}}
$$

the last series being uniformly and absolutely convergent for $0 \leqq \theta \leqq \pi$ and for all $|\xi| \geqq \rho>1$. Substituting (10) in (9)

$$
Q_{n}(z)=\sum_{m=1}^{\infty} \frac{\sigma_{n m}}{\xi^{m}},
$$

where

$$
\sigma_{n m}=\int_{0}^{\pi} P_{n}(\cos \theta) \sin (m \theta) d \theta .
$$

Since $\left|P_{n}(\cos \theta)\right| \leqq 1$ for $0 \leqq \theta \leqq \pi$ [5, Corollary 12.4.2] it follows that 


$$
\left|\sigma_{n m}\right| \leqq \int_{0}^{\pi}|\sin (m \theta)| d \theta=\int_{0}^{\pi} \sin \theta d \theta=2 .
$$

Again, setting $t=\cos \theta$,

$$
\sigma_{n m}=\int_{-1}^{1} P_{n}(t) \frac{\sin (m \arccos t)}{\left(1-t^{2}\right)^{1 / 2}} d t .
$$

Now $T_{m}(t)=\cos (m$ arc $\cos t)=$ Chebyshev polynomial of the first kind and

$$
\frac{\sin (m \arccos t)}{\left(1-t^{2}\right)^{1 / 2}}=\frac{1}{m} T_{m}{ }^{\prime}=U_{m-1}(t),
$$

where $U_{m-1}(t)$ is the Chebyshev polynomial of the second kind of degree $m-1$. Thus,

$$
\sigma_{n m}=\int_{-1}^{1} P_{n}(t) U_{m-1}(t) d t .
$$

From the orthogonality and symmetry of the Legendre polynomials, it follows that $\sigma_{n m}=0$ for $m-1<n$, i.e., for $m<n+1$ and $m=n+2, n+4, \cdots$. This establishes the lemma.

However, the following evaluation of $\sigma_{n m}$ is due to Heine [6, p. 311]:

$$
\begin{aligned}
\sigma_{n m} & =\int_{0}^{\pi} P_{n}(\cos \theta) \sin (m \theta) d \theta \\
& =\Gamma\left(\frac{m+n+1}{2}\right) \Gamma\left(\frac{m-n}{2}\right) / \Gamma\left(\frac{m-n+1}{2}\right) \Gamma\left(\frac{m+n+2}{2}\right)
\end{aligned}
$$

when $m>n$ and $m-n$ is odd, and the value is zero in all other cases.

2.2. Convergence of the Gauss-Legendre Quadrature. From Lemma 2, we have for $z$ on $\varepsilon_{\rho}$,

$$
\left|Q_{n}(z)\right| \leqq \sum_{k=n+1}^{\infty} \frac{2}{\rho_{k}}=\frac{2 \rho}{\rho^{2}-1} \rho^{-n} .
$$

Also, for $z \in \varepsilon_{\rho}$, it is easy to show $[5,12.4 .10]$ that

$$
\lim _{n \rightarrow \infty}\left|P_{n}(z)\right|^{1 / n}=\rho .
$$

That is, given $\epsilon>0$, there is an $N(\epsilon)$, such that for $n \geqq N(\epsilon)$,

$$
\left|P_{n}(z)\right| \geqq(\rho-\epsilon)^{n} .
$$

From (4), by selecting the contour as an ellipse $\varepsilon_{\rho}(\rho>1)$, it follows that

$$
\left|E_{G_{n}}(f)\right| \leqq \frac{1}{\pi} \int_{\varepsilon_{\rho}} \frac{|f(z)|\left|Q_{n}(z)\right| d s}{\left|P_{n}(z)\right|} \quad(|d z|=d s) .
$$

Employing (15) and (16), from (17) we obtain for $n \geqq N(\epsilon)$,

$$
\begin{aligned}
\left|E_{G_{n}}(f)\right| & \leqq\left(\frac{1}{\pi} \frac{2 \rho l\left(\varepsilon_{\rho}\right) M(\rho)}{\rho^{2}-1}\right) \rho^{-n}(\rho-\epsilon)^{-n} \\
& \leqq 2 K \frac{M(\rho)}{\rho^{2 n}}(1-\epsilon / \rho)^{-n}
\end{aligned}
$$


where $l\left(\varepsilon_{\rho}\right)=$ length of $\varepsilon_{\rho}$ and $K=l\left(\varepsilon_{\rho}\right) / \pi\left(\rho-\rho^{-1}\right)$.

We have thus established the following result:

THEOREM 1. Let $f \in A[-1,1]$ and be continuable analytically so as to be singlevalued and regular in the closed ellipse $\varepsilon_{\rho}$ whose foci are at $z= \pm 1$ and whose sum of semi-axes is $\rho(\rho>1)$. Then, given $\epsilon>0$, we have for $n \geqq N(\epsilon)$,

$$
\left|E_{G_{n}}(f)\right| \leqq 2 K M(\rho)(1-\epsilon / \rho)^{-n} / \rho^{2 n}
$$

where $M(\rho)=\max |f(z)|$ on $\varepsilon_{\rho}$.

A similar estimate for $E_{G_{n}}(f)$ has been given by Davis [2, Eq. (35)].

2.3. Error Estimates for the Gauss-Legendre Quadrature. Assume that $f(z)$ is regular in a sufficiently large domain $D$ of the $z$-plane containing the interval $[-1,1]$. In (4), choose the contour $L:|z|=R$ with sufficiently large $R$, in $D$. The asymptotic value of $Q_{n}(z) / P_{n}(z)$, for $|z| \rightarrow \infty$, can be obtained from

$$
\frac{Q_{n}(z)}{P_{n}(z)}=\frac{2^{2 n}(n !)^{4}}{(2 n) !(2 n+1) !} z^{-2 n-1}\left[1+\frac{2 n^{3}+3 n^{2}-n-1}{(2 n+3)(2 n-1)} z^{-2}+O\left(z^{-4}\right)\right]
$$

Taking only the first term of the above expansion for $Q_{n}(z) / P_{n}(z)$, there follows the following estimate for the error of the Gauss-Legendre quadrature.

Theorem 2. Let $f \in A[-1,1]$ and be continuable analytically so as to be singlevalued and regular in a suffciently large domain $D$ of the z-plane containing the interval $[-1,1]$ in its interior. Then, for sufficiently large $R$,

$$
\left|E_{G_{n}}(f)\right| \leqq 2 K_{n} M(R) / R^{2 n},
$$

where $K_{n}=2^{2 n}(n !)^{4} /(2 n) !(2 n+1) !$ and $M(R)=\max |f(z)|$ on $|z|=R$ in $D$.

2.4. Next, we consider the case when the integrand $f(z)$ has singularities in the $z$-plane. We shall consider the case when $f(z)$ has a simple pole at $z=z_{0}$.

In this case the evaluation of the contour integral (1) gives

$$
\frac{1}{2 \pi i} \int_{L} \frac{f(z) d z}{(z-t) P_{n}(z)}=\sum_{i=1}^{n} \frac{f\left(t_{i}\right)}{\left(t_{i}-t\right) P_{n}^{\prime}\left(t_{i}\right)}+\frac{f(t)}{P_{n}(t)}+\frac{\phi\left(z_{0}\right)}{\left(z_{0}-t\right) P_{n}\left(z_{0}\right)}
$$

where

$$
\phi\left(z_{0}\right)=\lim _{z \rightarrow z_{0}} \frac{d}{d z}\left[\left(z-z_{0}\right)^{2} f(z)\right]
$$

Therefore

$$
f(t)=\sum_{i=1}^{n} \frac{P_{n}(t)}{\left(t-t_{i}\right) P_{n}^{\prime}\left(t_{i}\right)} f\left(t_{i}\right)+\frac{\phi\left(z_{0}\right)}{P_{n}\left(z_{0}\right)} \frac{P_{n}(t)}{\left(t-z_{0}\right)}+\frac{1}{2 \pi i} \int_{L} \frac{f(z) P_{n}(t) d z}{(z-t) P_{n}(z)} .
$$

Integrating with respect to $t$ from -1 to 1 , and employing (5), there results the quadrature formula (3) with the error given by

$$
E_{G_{n}}(f)=\frac{1}{\pi i} \int_{L} \frac{f(z) Q_{n}(z) d z}{P_{n}(z)}-\frac{2 \phi\left(z_{0}\right) Q_{n}\left(z_{0}\right)}{P_{n}\left(z_{0}\right)} .
$$

As $|z| \rightarrow \infty$, the first term will tend to zero and the error in this case will be bounded by

$$
\left|E_{G_{n}}(f)\right| \leqq 2\left|\phi\left(z_{0}\right)\right|\left|Q_{n}\left(z_{0}\right) / P_{n}\left(z_{0}\right)\right|
$$


In case $\left|z_{0}\right|>1$, the upper bound tends to zero with increasing $n$, as follows from (19). But if $z_{0} \rightarrow 1$, the upper bound increases unboundedly.

3. The Gauss-Chebyshev Quadrature Formula. From the contour integral

$$
\frac{1}{2 \pi i} \int_{L} \frac{f(z) d z}{(z-t) T_{n}(z)}
$$

where $f(z)$ is regular within $L$, it is easily deduced that

$$
f(t)=\sum_{i=1}^{n} \frac{T_{n}(t)}{\left(t-t_{i}\right) T_{n}{ }^{\prime}\left(t_{i}\right)} f\left(t_{i}\right)+\frac{1}{2 \pi i} \int_{L} \frac{f(z) T_{n}(t) d z}{(z-t) T_{n}(z)},
$$

which is the Lagrange interpolation formula, with the error term, for $f(z)$ at the zeros of the Chebyshev polynomial $T_{n}(t)$ defined on $[-1,1]$.

Integrating both sides of $(23)$ on $[-1,1]$ with the weight-function $\left(1-t^{2}\right)^{-1 / 2}$, we obtain

$$
\int_{-1}^{1} \frac{f(t) d t}{\left(1-t^{2}\right)^{1 / 2}}=\sum_{i=1}^{n} \mu_{i} f\left(t_{i}\right)+\frac{1}{2 \pi i} \int_{L} \frac{f(z)}{T_{n}(z)}\left(\int_{-1}^{1} \frac{T_{n}(t) d t}{(z-t)\left(1-t^{2}\right)^{1 / 2}}\right) d z
$$

This is the Gauss-Chebyshev quadrature formula of order $n$ on $[-1,1]$. The abscissas and the weights are given respectively by

$$
t_{i}=\cos ((2 i-1) \pi / 2 n), \quad \mu_{i}=\pi / n, \quad i=1, \cdots, n .
$$

Define

$$
Q_{n}^{*}(z)=\frac{1}{2} \int_{-1}^{1} \frac{T_{n}(t) d t}{\left(1-t^{2}\right)^{1 / 2}(z-t)}
$$

then $Q_{n}{ }^{*}(z)$ is a single-valued analytic function for all $z$ in the plane with the interval $[-1,1]$ deleted. $Q_{n}{ }^{*}(z)$ corresponds to $Q_{n}(z)$, the Legendre function of the second kind.

From (24), in view of (25), the error of the Gauss-Chebyshev quadrature formula can be put in the form

$$
E_{T_{n}}(f)=\frac{1}{\pi i} \int_{L} \frac{f(z) Q_{n}^{*}(z) d z}{T_{n}(z)} .
$$

3.1. Lemmas for $Q_{n}{ }^{*}(z)$. In the following we prove two lemmas for the function $Q_{n}{ }^{*}(z)$ and subsequently employ them to derive error estimates for the GaussChebyshev quadrature formula.

The following lemma gives a simple representation for $Q_{n}{ }^{*}(z)$ on $\varepsilon_{\rho}$.

Lemma 3. For $z \in \varepsilon_{\rho}$

$$
Q_{n}^{*}(z)=\sum_{m=n+1}^{\infty} \frac{\sigma_{n m}^{*}}{\xi^{m}}
$$

where

$$
\sigma_{n m}^{*}=\pi \quad \text { for } n=0,1,2, \cdots, \quad m=n+1, n+3, \cdots .
$$

$A l s o, Q_{n}{ }^{*}(z)$ can be expressed in the closed form 


$$
Q_{n}{ }^{*}(z)=\frac{\pi}{2\left(z^{2}-1\right)^{1 / 2}\left(z \pm\left(z^{2}-1\right)^{1 / 2}\right)^{n}}, \quad z \in \varepsilon_{\rho},
$$

where the sign is chosen so that $\left|z \pm\left(z^{2}-1\right)^{1 / 2}\right|>1$.

Proof. Transforming (25) to the $\xi$-plane, setting $t=\cos \theta$, and proceeding exactly as in Lemma 1 , we obtain

$$
Q_{n}^{*}(z)=\sum_{m=1}^{\infty} \frac{\sigma_{n m}^{*}}{\xi^{m}}
$$

where

$$
\sigma_{n m}^{*}=\int_{0}^{\pi} \frac{T_{n}(\cos \theta) \sin (m \theta)}{\sin \theta} d \theta .
$$

Setting again $t=\cos \theta$,

$$
\sigma_{n m}^{*}=\int_{-1}^{1} \frac{T_{n}(t) U_{m-1}(t) d t}{\left(1-t^{2}\right)^{1 / 2}} .
$$

Owing to orthogonality with respect to the weight $\left(1-t^{2}\right)^{-1 / 2}$ and the symmetry of the Chebyshev polynomials $U_{m}(t)$, it follows that $\sigma_{n m}^{*}=0$ for $m-1<n$ and $m=n+2, n+4, \cdots$. Since $T_{n}(\cos \theta)=\cos (n \theta),(29)$ gives

$$
\sigma_{n m}^{*}=\frac{1}{2} \int_{0}^{\pi} \frac{\sin (m+n) \theta+\sin (m-n) \theta}{\sin \theta} d \theta,
$$

where $m=n+1, n+3, \cdots$.

Futting $m=n+2 k+1$, where $k=0,1,2, \cdots$,

$$
\begin{aligned}
\sigma_{n m}^{*} & =\frac{1}{2} \int_{0}^{\pi} \frac{\sin (2 n+2 k+1) \theta+\sin (2 k+1) \theta}{\sin \theta} d \theta \\
& =\frac{1}{2}(\pi+\pi)=\pi
\end{aligned}
$$

for $n=0,1,2, \cdots, m=n+1, n+3, \cdots$.

This result corresponds to Heine's evaluation of $\sigma_{n m}$ in the case of the Legendre function $Q_{n}(z)$ indicated at the end of Lemma 2. However, in this case, $Q_{n}{ }^{*}(z)$ can be obtained in a closed form.

From (27), we have for $z \in \varepsilon_{\rho}$,

$$
Q_{n}^{*}(z)=\frac{\pi}{\xi^{n+1}} \sum_{m=0}^{\infty} \xi^{-2 m}=\frac{\pi}{\xi^{n}}\left(\xi-\xi^{-1}\right)^{-1} .
$$

$\left(27^{\prime}\right)$ now follows by observing that $\xi=z \pm\left(z^{2}-1\right)^{1 / 2}$. This proves the lemma.

The following lemma provides an expansion for $Q_{n}{ }^{*}(z)$, similar to that for $Q_{n}(z)$ [6, p. 288].

LemMa 4. For $|z|>1$,

$$
Q_{n}^{*}(z)=\sum_{m=n}^{\infty} \frac{\sigma_{n m}^{* *}}{z^{m+1}}
$$

where 


$$
\sigma_{n m}^{* *}=\frac{\pi}{2^{m+1}}\left(\begin{array}{c}
m \\
(m-n) / 2
\end{array}\right),
$$

$n=0,1,2, \cdots, m=n, n+2, \cdots$.

Proof. We start with (25). For $|z|>1$,

$$
Q_{n}{ }^{*}(z)=\frac{1}{2} \int_{-1}^{1} \frac{T_{n}(t)}{\left(1-t^{2}\right)^{1 / 2}}\left[\sum_{m=0}^{\infty} \frac{t^{m}}{z^{m+1}}\right] d t=\sum_{m=0}^{\infty} \frac{\sigma_{n m}^{* *}}{z^{m+1}},
$$

where

$$
\sigma_{n m}^{* *}=\frac{1}{2} \int_{-1}^{1} \frac{T_{n}(t) t^{m} d t}{\left(1-t^{2}\right)^{1 / 2}}
$$

Since

$$
t^{m}=2^{-m+1} \sum_{k=0}^{[m / 2]}\left(\begin{array}{c}
m \\
k
\end{array}\right) T_{m-2 k},
$$

where the prime on the summation sign indicates that the last term is to be halved when $m$ is even. Substituting (33) in (32) and using the orthogonality of the Chebyshev polynomials,

$$
\begin{aligned}
\sigma_{n m}^{* *} & =\sum_{k=0}^{[m / 2]}\left(\begin{array}{c}
m \\
k
\end{array}\right)\left(2^{-m} \int_{-1}^{1} \frac{T_{n}(t) T_{m-2 k}(t) d t}{\left(1-t^{2}\right)^{1 / 2}}\right) \\
& =\sum_{k=0}^{[m / 2]}\left(\begin{array}{c}
m \\
k
\end{array}\right) 2^{-m} \frac{\pi}{2} \delta_{n, m-2 k} \\
& =\frac{\pi}{2^{m+1}}\left(\begin{array}{c}
m \\
(m-n) / 2
\end{array}\right) .
\end{aligned}
$$

From the orthogonality and symmetry of the Chebyshev polynomials, it follows from (32) that $\sigma_{n m}^{* *}=0$, if $m<n$ and $m=n+1, n+3, \cdots$. This proves the lemma.

The following are immediate consequences of Lemma 4.

Corollary 1. For all sufficiently large $|z|$,

$$
\left|Q_{n}^{*}(z)\right| \leqq\left(\pi / 2^{n+1}\right)|z|^{-n-1} .
$$

Corollary 2. For $|z|>1$,

$$
\left(Q_{n}^{*}(z) / T_{n}(z)\right)=\left(\pi / 2^{2 n}\right) z^{-2 n-1}\left[1+\frac{1}{2} z^{-2}+\cdots\right]
$$

for $n \geqq 1$; if $n=0$, the right side is to be divided by 2 .

3.2. Convergence of the Gauss-Chebyshev Quadrature. From Lemma 3, it follows that on $\varepsilon_{\rho}$,

$$
\left|Q_{n}^{*}(z)\right| \leqq\left(\pi / \rho^{n+1}\right)\left(1-\rho^{-2}\right)^{-1} .
$$

Also, for $z \in \varepsilon_{\rho}$, it is easy to verify $[5,4.4 .5]$ that

$$
\lim _{n \rightarrow \infty}\left|T_{n}(z)\right|^{1 / n}=\rho .
$$

That is, given $\epsilon>0$, there is an $N(\epsilon)$ such that for $n \geqq N(\epsilon)$, 


$$
\left|T_{n}(z)\right| \geqq(\rho-\epsilon)^{n} .
$$

From (26), by selecting the contour as an ellipse $\varepsilon_{\rho}(\rho>1)$, we obtain

$$
\left|E_{T_{n}}(f)\right| \leqq \frac{1}{\pi} \int_{\varepsilon_{\rho}} \frac{|f(z)|\left|Q_{n}^{*}(z)\right|}{\left|T_{n}(z)\right|} d s .
$$

Employing (36) and (37), from (38) follows the following estimate for the error of the Gauss-Chebyshev quadrature formula.

TheOREM 3. Let $f$ satisfy the regularity conditions of Theorem 1 and let $M(\rho)$ and $K$ be as defined there. Then, for every $\epsilon>0$, we have for $n \geqq N(\epsilon)$,

$$
\left|E_{T_{n}}(f)\right| \leqq \pi K\left(M(\rho) / \rho^{2 n}\right)(1-\epsilon / \rho)^{-n} .
$$

3.3. Error Estimates for the Gauss-Chebyshev Quadrature. Let $f(z)$ be regular in a sufficiently large domain $D$ of the $z$-plane containing the interval $[-1,1]$ in its interior. In (26), taking $L:|z|=R$ with sufficiently large $R$ and taking only the first term of the asymptotic expansion for $Q_{n}{ }^{*}(z) / T_{n}(z)$ given by (35), the following estimate of error of the Gauss-Chebyshev quadrature follows.

TheOREM 4. Let $f$ satisfy the regularity conditions of Theorem 2. Then, for sufficiently large $R$,

$$
\left|E_{T_{n}}(f)\right| \leqq\left(\pi / 2^{2 n-1}\right) M(R) / R^{2 n} .
$$

An extension to the case of the integrand $f(z)$ having simple poles can be carried out as in Section 2.4 .

3.4. The error estimates (20) and (40) are simple to obtain and will not be unduly pessimistic, but hold for functions having a sufficiently large circle of analyticity. We can, however, obtain simple estimates of error for the Gauss-Chebyshev quadrature formula for all functions analytic on $[-1,1]$.

Let $f$ be analytic on $[-1,1]$. Then, for some $\rho>1, f$ can be continued analytically so as to be regular in the closed ellipse $\varepsilon_{\rho}$. Taking the ellipse $\varepsilon_{\rho}$ for the contour in (26), by virtue of $\left(27^{\prime}\right)$, the error of the Gauss-Chebyshev quadrature can be put in the form

$$
E_{T_{n}}(f)=\frac{1}{2 i} \int_{\varepsilon_{\rho}} \frac{f(z) d z}{\left(z^{2}-1\right)^{1 / 2}\left(z \pm\left(z^{2}-1\right)^{1 / 2}\right)^{n} T_{n}(z)} .
$$

Since on $\varepsilon_{\rho}$,

$$
T_{n}(z)=\frac{1}{2}\left(\xi^{n}+\xi^{-n}\right)
$$

(41), on transformation to the $\xi$-plane, gives

$$
E_{T_{n}}(f)=-i \int_{C_{\rho}} \frac{f\left[\frac{1}{2}\left(\xi+\xi^{-1}\right)\right] d \xi}{\left(\xi^{n}+\xi^{-n}\right) \xi^{n+1}} \quad\left(C_{\rho}:|\xi|=\rho\right) .
$$

From (43) follows the following estimate of error:

Theorem 5. Let $f \in A[-1,1]$ and let $\rho>1$ be such that $f(z)$ is regular in the closure of $\varepsilon_{\rho}$. Then

$$
\left|E_{T_{n}}(f)\right| \leqq 2 \pi M(\rho) /\left(\rho^{2 n}-1\right),
$$

where $M(\rho)=\max |f(z)|$ on $\varepsilon_{\rho}$ (or equivalently on $C_{\rho}$ ). 
Remark. For fixed $n$ and varying $\rho$, a "least conservative" upper bound (44) can be established for some $\rho^{*}\left(1<\rho^{*} \leqq \rho\right)$. Observe, however, that in case the integrand $f(z)$ is entire, $\rho^{*}$ will be a value of $\rho$ for which $M(\rho) /\left(\rho^{2 n}-1\right)$ is a minimum. Similar remarks apply to the estimates (20) and (40).

Acknowledgements. We are grateful to the referee for his most valuable comments which helped us in bringing the paper to its present form. We wish to thank Professor Philip J. Davis for communicating this paper, and Professor L. Collatz for his helpful comments.

Department of Mathematics

Indian Institute of Technology

Hauz Khas, New Delhi-29

India

1. P. J. Davis \& P. Rabinowitz, "On the estimation of quadrature errors for analytic functions," $M T A C$, v. 8, 1954, pp. 193-203. MR 16, 404.

2. P. J. DAvis, "On the numerical integration of periodic analytic functions," On Numerical Approximation, R. E. Langer, Editor, Univ. of Wisconsin Press, Madison, Wis., 1959, pp. 45-59. 3. J. McNAMEe, "Error-bounds for the evaluation of integrals by the Euler-Maclaurin formula and by Gauss-type formulae," Math. Comp., v. 18, 1964, pp. 368-381. MR 32 \#3264.

4. W. BARRETT, "Convergence properties of Gaussian quadrature formulae," Comput. J., v. 3, 1960/1961, pp. 272-277. MR 23 \#B1117.

5. P. J. DAvis, Interpolation and Approximation, Blaisdell, New York, 1963. MR 28 \#393.

6. E. T. Copson, Theory of Functions of a Complex Variable, Oxford Univ. Press, Oxford, 1935. 